\title{
A Note on Invariants and Entanglements
}

\author{
Sergio Albeveriof and Shao-Ming Feif \\ Institut für Angewandte Mathematik, Universität Bonn, D-53115 Bonn
}

\begin{abstract}
The quantum entanglements are studied in terms of the invariants under local unitary transformations. A generalized formula of concurrence for $N$-dimensional quantum systems is presented. This generalized concurrence has potential applications in studying separability and calculating entanglement of formation for high dimensional mixed quantum states.
\end{abstract}

PACS numbers: 03.65.Bz, 89.70.+c

\footnotetext{
${ }^{1}$ SFB 256; BiBoS; CERFIM (Locarno); Acc.Arch., USI (Mendrisio)

${ }^{2}$ Institute of Applied Mathematics, Chinese Academy of Science, Beijing.
} 
Quantum entanglement is tightly related to the foundations of quantum mechanics, particularly to quantum nonseparability and the violation of Bell's inequalities [1]. It has also been playing important roles in communication, information processing and quantum computing [2], such as in the investigation of quantum teleportation [3, 4], dense coding [5], decoherence in quantum computers and the evaluation of quantum cryptographic schemes [6]. To quantify entanglement, a well justified and mathematically tractable measure is needed. A number of entanglement measures such as the entanglement of formation and distillation [7, 8, 9], negativity [10, 11], von Neumann entropy and relative entropy [9, 12] have been proposed for bipartite states $[6,8,12-15]$ and some of their relations have been discussed [16], though most proposed measures of entanglement involve extremizations which are difficult to handle analytically.

The entanglement of formation [7] is intended to quantify the amount of quantum communication required to create a given state. For the entanglement of a pair of qubits, it has been shown that the entanglement of formation can be expressed as a monotonically increasing function of the "concurrence" $C$. This function ranges from 0 to 1 as $C$ goes from 0 to 1 , so that one can take the concurrence as a measure of entanglement in its own right [14]. From the expression of $C$, which is much simpler than the definition of entanglement of formation, the entanglement of formation for mixed states of a pair of qubits is calculated [14]. Nevertheless so far no explicit analytic formulae for entanglement of formation have been found for systems larger than a pair of qubits (the case being special in many ways [15]), although entanglement of formation is defined for arbitrary dimension.

In fact, as the degree of entanglement will neither increase nor decrease under local unitary transformations on a subquantum system, the measure of entanglement must be an invariant of local unitary transformations. In this note we describe entanglements from the view of this kind of invariants. A generalized explicit formula of concurrence for high dimensional bipartite systems is derived from the relations among these invariants.

Consider the case of quantum systems with an $N$-dimensional complex Hilbert space $\mathcal{H}$. Let $e_{i}, i=1, \ldots, N$, be an orthonormal the basis of the Hilbert space. A general pure state 
of two $N$-dimensional quantum systems is of the form,

$$
\mid \Psi>=\sum_{i, j=1}^{N} a_{i j} e_{i} \otimes e_{j}, \quad a_{i j} \in \mathbb{C}
$$

with normalization

$$
\sum_{i, j=1}^{N} a_{i j} a_{i j}^{*}=1
$$

The entanglement of formation is given by

$$
E(\mid \Psi>)=-\operatorname{Tr}\left(\rho_{0} \log _{2} \rho_{0}\right)
$$

where $\rho_{0}$ is the partial trace of $|\Psi><\Psi|$ over one of the subsystems. For $N=2$, the state $\mid \Psi>$ is factorizable into single-qubit (unentangled) states if and only if $a_{11} a_{22}=a_{21} a_{12}$. It is shown that

$$
C=2\left|a_{11} a_{22}-a_{12} a_{21}\right|
$$

which ranges from 0 to 1 , is a plausible measure of the degree of entanglement. This is taken to be the definition of concurrence for a pure state of two qubits [14].

Let $U$ denote the unitary transformations on the Hilbert space $\mathcal{H}$, such that

$$
U e_{i} \mapsto \sum_{j=1}^{N} b_{i j} e_{j}, \quad b_{i j} \in \mathbb{C}
$$

and

$$
\sum_{j=1}^{N} b_{i j} b_{k j}^{*}=\delta_{i k}
$$

We call a quantity an invariant associated with the state $\mid \Psi>$ if it is invariant under the local unitary transformations of $U \otimes U$. Let $A$ denote the matrix given by $(A)_{i j}=a_{i j}$. Hence $\rho_{0}=A A^{\dagger}$. By generalizing the results of analysis on invariants for qubits [17], we can show that the following quantities are invariants under local unitary transformations:

$$
I_{\alpha}=\operatorname{Tr}\left(A A^{\dagger}\right)^{\alpha+1}, \quad \alpha=0,1, \ldots, N-1 .
$$

Among these invariants,

$$
I_{0}=\operatorname{Tr}\left(A A^{\dagger}\right)=\sum_{i, j=1}^{N} a_{i j} a_{i j}^{*}
$$

and

$$
I_{1}=\operatorname{Tr}\left[\left(A A^{\dagger}\right)^{2}\right]=\sum_{i, j, k, m=1}^{N} a_{i k} a_{i m}^{*} a_{j m} a_{j k}^{*}
$$


are of particular importance. $I_{0}$ is in fact the normalization condition (2). $I_{1}$ is a biquadratic form of the coefficients $a_{i j}$.

Polynomials in $I_{0}$ and $I_{1}$ are obviously also invariants. Among them the quantity $\frac{N}{N-1}\left(I_{0}^{2}-I_{1}\right)$ is of special significance:

$$
\begin{aligned}
\frac{N}{N-1}\left(I_{0}^{2}-I_{1}\right) & =\frac{N}{N-1} \sum_{i, j, k, m=1}^{N}\left(a_{i k} a_{i k}^{*} a_{j m} a_{j m}^{*}-a_{i k} a_{i m}^{*} a_{j m} a_{j k}^{*}\right) \\
& =\frac{N}{2(N-1)} \sum_{i, j, k, m=1}^{N}\left(a_{i k} a_{j m}-a_{i m} a_{j k}\right)\left(a_{i k}^{*} a_{j m}^{*}-a_{i m}^{*} a_{j k}^{*}\right) \\
& =\frac{N}{2(N-1)} \sum_{i, j, k, m=1}^{N}\left|a_{i k} a_{j m}-a_{i m} a_{j k}\right|^{2} .
\end{aligned}
$$

For the case of $N=2$, we see that the square root of $\frac{N}{N-1}\left(I_{0}^{2}-I_{1}\right)$ is just the concurrence (3). For general $N$, we see that $\frac{N}{N-1}\left(I_{0}^{2}-I_{1}\right)$ is positive definite and takes values from 0 to 1 . It takes the value zero when the state $|\Psi\rangle$ is factorizable, $a_{i j}=a_{i} b_{j}$ for some $a_{i}$, $b_{j} \in \mathbb{C}, i, j=1, \ldots, N$, and one when $\mid \Psi>$ is maximally entangled, e.g., $a_{i i}=1 / \sqrt{N}$, $a_{i j}=0$ for $i \neq j$. The term $\left|a_{i k} a_{j m}-a_{i m} a_{j k}\right|$ stands for the contribution of the terms $a_{i k} e_{i} \otimes e_{k}+a_{j m} e_{j} \otimes e_{m}+a_{i m} e_{i} \otimes e_{m}+a_{j k} e_{j} \otimes e_{k}$ in $\mid \Psi>$ to the "degree of entanglement". When $a_{i k} a_{j m}-a_{i m} a_{j k}=0$, these terms can be written in a factorized form $\left(a_{1} e_{i}+a_{2} e_{j}\right) \otimes\left(b_{1} e_{k}+b_{2} e_{m}\right)$ for some $a_{1}, b_{1}, a_{2}, b_{2} \in \mathbb{C}$ and give zero contribution to the entanglement. When $a_{i k} a_{j m}=1$ (resp. $a_{i m} a_{j k}=1$ ) and $a_{i m} a_{j k}=0$ (resp. $a_{i k} a_{j m}=0$ ), they give the maximal contribution to the entanglement. If $\mid \Psi>$ is unentangled, i.e., $\mid \Psi>=\left(\sum_{i=1}^{N} a_{i} e_{i}\right) \otimes\left(\sum_{j=1}^{N} b_{j} e_{j}\right), a_{i j}=a_{i} b_{j}$, then $\left|a_{i k} a_{j m}-a_{i m} a_{j k}\right|=0, \forall i, j, k, m=1, \ldots, N$, and we have $I_{0}^{2}-I_{1}=0$. All entangled states have at least one of the terms $\left|a_{i k} a_{j m}-a_{i m} a_{j k}\right| \neq 0$, hence they have non zero $I_{0}^{2}-I_{1}$.

Therefore $\frac{N}{N-1}\left(I_{0}^{2}-I_{1}\right)$ could be a suitable candidate for the measure of entanglement of two $N$ dimensional quantum systems $\mid \Psi>$ in some sense. In accord with the definition of concurrence for two qubits [14, we take the square root of it to be the generalized concurrence:

$$
C_{N}=\sqrt{\frac{N}{N-1}\left(I_{0}^{2}-I_{1}\right)}=\sqrt{\frac{N}{2(N-1)} \sum_{i, j, k, m=1}^{N}\left|a_{i k} a_{j m}-a_{i m} a_{j k}\right|^{2}} .
$$

It is seen that $C_{2}=C$. The factor $\frac{N}{N-1}$ in (8) is just so chosen such that the maximal value of $C_{N}$ is scaled to be one.

To understand the relations between the generalized concurrence $C_{N}$ and the entan- 
glement of formation $E(\mid \Psi>$ ), we now study the properties of the invariants. In terms of the Schmidt decomposition, a given $\mid \Psi>$ can always be written in the form, in some orthonormal basis $\left\{e_{i}\right\}, i=1, \ldots, N$,

$$
\mid \Psi>=\sum_{i=1}^{N} \sqrt{\Lambda_{i}} e_{i} \otimes e_{i},
$$

where $\sum_{i=1}^{N} \Lambda_{i}=1, \Lambda_{i} \geq 0$. The matrix $\rho_{0}$ is then of the form $\rho_{0}=A A^{\dagger}=\operatorname{diag}\left(\Lambda_{1}, \ldots \Lambda_{N}\right)$. The entanglement of formation of $\mid \Psi>$ is given by

$$
E(\mid \Psi>)=-\sum_{i=1}^{N} \Lambda_{i} \log _{2} \Lambda_{i}
$$

The invariants are then of the form

$$
I_{\alpha}=\sum_{i=1}^{N} \Lambda_{i}^{\alpha+1}, \quad \alpha=0, \ldots, N-1 .
$$

First we note that

$$
I_{1}=\sum_{i=1}^{N} \Lambda_{i}^{2}=I_{0}^{2}-\sum_{i \neq j}^{N} \Lambda_{i} \Lambda_{j} .
$$

Therefore $C_{N}=0$ implies that $\sum_{i \neq j}^{N} \Lambda_{i} \Lambda_{j}=0$. As $\Lambda_{i} \geq 0$ and $\sum_{i=1}^{N} \Lambda_{i}=1$, we have that only one $\Lambda$, say $\Lambda_{1}$, equals to 1 and the rest be zero. In this case $I_{\alpha}=I_{0}^{\alpha+1}, \alpha=1, \ldots, N-1$, and $E(\Psi)=0$. That is $I_{0}^{2}-I_{1}=0$ implies $E(\Psi)=0$.

If $C_{N}=1$, we have $\sum_{i \neq j}^{N} \Lambda_{i} \Lambda_{j}=\frac{N-1}{N}$, which is equivalent to the condition $\sum_{i=1}^{N} \Lambda_{i}^{2}=$ $1 / N$, according to the normalization $\sum_{i=1}^{N} \Lambda_{i}=1$. Equation $\sum_{i=1}^{N} \Lambda_{i}^{2}=1 / N$ describes a $N$ - 1 -dimensional sphere in $\mathbb{R}^{N}$ with radius $1 / \sqrt{N}$, whereas $\sum_{i=1}^{N} \Lambda_{i}=1$ is a hyperplane in $\mathbb{R}^{N}$. These geometrical objects have only one contact point at $\Lambda_{i}=1 / N$ for $\Lambda_{i} \geq 0$, $i=1, \ldots, N$. Therefore $C_{N}=1$ implies that $E(\Psi)=\sum_{i=1}^{N} \Lambda_{i} \log _{2} \Lambda_{i}=1$.

Therefore the invariant $I_{0}^{2}-I_{1}$ and hence the generalized concurrence $C_{N}$ characterizes the properties of entanglement in some way. However we remark that the above properties of $C_{N}$ do not mean that $C_{N}$ is in general a suitable measure for general $N$-dimensional bipartite quantum pure states. It can however be shown that when the matrix $A A^{\dagger}$ has only two different nonzero eigenvalues, the entanglement of formation is a monotonically increasing function of $C_{N}$, thus $C_{N}$ can indeed be used as a measure of entanglement in this case. 
In fact one can show that the eigenvalue equation for the $\Lambda_{i}, i=1, \ldots, N$, has the form:

$$
\sum_{k=1}^{N}(-\Lambda)^{k} c_{k}=0
$$

with $c_{k}$ polynormials in the invariants $I_{0}, \ldots, I_{N-1}$. E.g.,

$$
\begin{aligned}
& c_{N}=1, \quad c_{N-1}=I_{0}, \quad c_{N-2}=\frac{1}{2}\left(I_{0}^{2}-I_{1}\right), \\
& c_{N-3}=\frac{1}{6}\left(I_{0}^{3}+2 I_{2}-3 I_{0} I_{1}\right), \\
& c_{N-4}=\frac{1}{24}\left(I_{0}^{4}-6 I_{0}^{2} I_{1}+8 I_{0} I_{2}+3 I_{1}^{2}-6 I_{3}\right) .
\end{aligned}
$$

As $I_{0}$ is normalized to be one, the coefficient $c_{N-2}=\left(I_{0}^{2}-I_{1}\right) / 2$ is the first non trivial one in the eigenvalue equation of $\Lambda$. To see the role of $I_{0}^{2}-I_{1}$ in the equation, let us take $N=3$. We have then

$$
\begin{aligned}
& \Lambda_{1}=\frac{1}{3}+\frac{2}{3} \cos \frac{\phi}{3} \sqrt{1-C_{3}^{2}} \\
& \Lambda_{2}=\frac{1}{3}-\frac{1}{3}\left(\cos \frac{\phi}{3}+\sqrt{3} \sin \frac{\phi}{3}\right) \sqrt{1-C_{3}^{2}} \\
& \Lambda_{3}=\frac{1}{3}-\frac{1}{3}\left(\cos \frac{\phi}{3}-\sqrt{3} \sin \frac{\phi}{3}\right) \sqrt{1-C_{3}^{2}}
\end{aligned}
$$

where $C_{3}^{2}=\left(C_{3}\right)^{2}=\frac{3}{2}\left(I_{0}^{2}-I_{1}\right), \phi=\arctan \frac{\sqrt{B_{2}}}{B_{1}}, B_{1}=2-9 c_{1}+27 c_{0}, B_{2}=\left|4\left(3 c_{1}-1\right)^{3}+B_{1}\right|$, $c_{1}, c_{2}$ as in (10). For $C_{3}=0$ (resp. $C_{3}=1$ ) we have $\Lambda_{1}=1, \Lambda_{2}=\Lambda_{3}=0$ (resp. $\Lambda_{1}=\Lambda_{2}=\Lambda_{3}=1$ ). Nevertheless, the entanglement of formation (9) is not a monotonically increasing function of $C_{3}$. This is different to the case $N=2$, where according to the condition $\sum_{i=1}^{N} \Lambda_{i}=1$, there is only one independent eigenvalue of $\rho_{0}$.

The generalized concurrence (8) is useful in finding the necessary and sufficient conditions for the separability of mixed states and in caculating the entanglement of formation for some classes of mixed desity matrices. Due to recent works by Peres [18] and Horodecki et al [19] there exist a simple criterion allowing one to judge, whether a given density matrix $\rho$, representing a $2 \times 2$ or $2 \times 3$ composite system, is separable. Nevertheless, the general problem of finding sufficient and necessary conditions for separability in higher dimensions remains open (see e.g. 20, 21] and references therein). A general condition for separability of a quantum state could in principle be obtained from the measure of entanglement. However most proposed measures of entanglement involve extremizations which are difficult to handle analytically. For instance, the "entanglement of formation" [0], is defined for arbitrary 
dimension, but so far no explicit analytic formulae for entanglement of formation have been found for systems larger than a pair of qubits (spin- $\frac{1}{2}$ particles). As applications of the generalized concurrence, we have presented the necessary and sufficient conditions for the separability of high dimensional rank two mixed states [22]. Let $\rho$ be a rank two state in $\mathcal{H} \otimes \mathcal{H}$, with $\left|E_{1}\right\rangle,\left|E_{2}\right\rangle$ being its two orthonormal eigenvectors corresponding to the two nonzero eigenvalues:

$$
\rho=p\left|E_{1}\right\rangle\left\langle E_{1}|+q| E_{2}\right\rangle\left\langle E_{2}\right|
$$

where $q=1-p \in(0,1)$. Generally $\left|E_{k}\right\rangle=\sum_{i, j=1}^{N} a_{i j}^{k} e_{i} \otimes e_{j}, a_{i j}^{k} \in \mathbb{C}$, with normalization $\sum_{i, j=1}^{N} a_{i j}^{k}\left(a_{i j}^{k}\right)^{*}=1, k=1,2$. With the notations:

$$
\begin{aligned}
& \alpha_{i j}^{k l}=a_{i j}^{2} a_{k l}^{2}-a_{i l}^{2} a_{k j}^{2}, \quad \gamma_{i j}^{k l}=a_{i j}^{1} a_{k l}^{1}-a_{i l}^{1} a_{k j}^{1} \\
& \beta_{i j}^{k l}=a_{i j}^{1} a_{k l}^{2}+a_{i j}^{2} a_{k l}^{1}-a_{i l}^{2} a_{k j}^{1}-a_{i l}^{1} a_{k j}^{2},
\end{aligned}
$$

it is shown that $\rho$ is separable if and only if there is $\theta \in \mathbb{R}$ such that

$$
\begin{gathered}
\gamma_{i j}^{k l}=e^{i \theta}\left(1-p^{{ }^{1}}\right) \alpha_{i j}^{k l}, \\
\beta_{i j}^{k l} \alpha_{m n}^{k l}=\alpha_{i j}^{k l} \beta_{m n}^{k l}, \quad \forall i, j, k, l, m, n ;
\end{gathered}
$$

and

$$
\frac{\mu_{2}\left(1+\left|\mu_{1}\right|^{2}\right)}{z-\mu_{1} \mu_{2} \bar{z}} \in[0,1]
$$

where $z=e^{i \theta} \bar{z}, z=\mu_{2}-\mu_{1} \neq 0, \mu_{1}$ and $\mu_{2}$ are the roots of the equation $\alpha_{i j}^{k l} \lambda^{2}+\beta_{i j}^{k l} \lambda+\gamma_{i j}^{k l}=0$, for some $i, j, k, l$ such that $\alpha_{i j}^{k l} \neq 0$. This criterion allows one to judge the separability of $\rho$ by simply calculating its two orthonormal eigenvectors. Here by using the theorem 1 in [23], a simple and effective alternative criterion, the negative-partial-trace-criterion, can be also applied in this case, though it is not sufficient for general high dimensional case ${ }^{5}$.

In [24] we have shown that if $A A^{\dagger}$ has only two non-zero eigenvalues, the entanglement of formation of the corresponding pure state is a monotonically increasing function of the generalized concurrence. From this the entanglement of formation for a class of $16 \times 16$ mixed density matrices is calculated.

The above approach can be extended to the case of multiquantum (particle) systems. We consider now the entanglement of three $N$ dimensional quantum systems. A general

\footnotetext{
${ }^{3}$ We would like to thank the referees for introducing us the reference
} 
quantum system is then of the form,

$$
\Psi_{3}=\sum_{i, j, k=1}^{N} a_{i j k} e_{i} \otimes e_{j} \otimes e_{k}
$$

where $a_{i j k} \in \mathbb{C}$ and $\sum_{i, j, k=1}^{N} a_{i j k} a_{i j k}^{*}=1$. We have one quadratic and three biquadratic invariants:

$$
\begin{array}{ll}
I_{0}=\sum_{i, j, k=1}^{N} a_{i j k} a_{i j k}^{*}, & I_{1}=\sum a_{i j k} a_{i j m}^{*} a_{p q m} a_{p q k}^{*}, \\
I_{2}=\sum a_{i k j} a_{i m j}^{*} a_{p m q} a_{p k q}^{*}, & I_{3}=\sum a_{k i j} a_{m i j}^{*} a_{m p q} a_{k p q}^{*} .
\end{array}
$$

$I_{1}$ is associated with the exchange of the third sub index. The corresponding contribution to entanglement is given by

$$
\begin{aligned}
I_{0}^{2}-I_{1} & =\sum a_{i j k} a_{p q m}\left(a_{i j k}^{*} a_{p q m}^{*}-a_{i j m}^{*} a_{p q k}^{*}\right) \\
& =\frac{1}{2} \sum\left(a_{i j k} a_{p q m}-a_{i j m} a_{p q k}\right)\left(a_{i j k}^{*} a_{p q m}^{*}-a_{i j m}^{*} a_{p q k}^{*}\right) \\
& =\frac{1}{2} \sum\left|a_{i j k} a_{p q m}-a_{i j m} a_{p q k}\right|^{2} .
\end{aligned}
$$

Similarly we have

$$
\begin{aligned}
I_{0}^{2}-I_{2} & =\frac{1}{2} \sum\left|a_{i j k} a_{p q m}-a_{i q k} a_{p j m}\right|^{2}, \\
I_{0}^{2}-I_{3} & =\frac{1}{2} \sum\left|a_{i j k} a_{p q m}-a_{p j k} a_{i q m}\right|^{2} .
\end{aligned}
$$

A generalized concurrence can be defined to be

$$
\begin{aligned}
C_{N}^{3} & =\sqrt{\frac{N}{3(N-1)}\left(3 I_{0}^{2}-I_{1}-I_{2}-I_{3}\right)} \\
& =\sqrt{\frac{N}{6(N-1)} \sum\left(\left|a_{i j k} a_{p q m}-a_{i j m} a_{p q k}\right|^{2}+\left|a_{i j k} a_{p q m}-a_{i q k} a_{p j m}\right|^{2}+\left|a_{i j k} a_{p q m}-a_{p j k} a_{i q m}\right|^{2}\right.} .
\end{aligned}
$$

It is clear that $C_{N}^{3}$ is zero when $\Psi_{3}$ is factorizable, i.e., $a_{i j k}=a_{i} b_{j} c_{k}$ for some $a_{i}, b_{j}, c_{k} \in \mathbb{C}$. For a maximally entangled state like $a_{i i i}=\frac{1}{\sqrt{N}}, i=1, \ldots, N$, and the rest $a_{i j k}$ being zero, we get $C_{N}^{3}=1$. Nevertheless, when one quantum system is separated from the other two, e.g., $a_{i j k}=a_{i j} b_{k}$ for some $a_{i j}, b_{k} \in \mathbb{C}, C_{N}^{3}$ is not zero, as the three quantum systems still have some degree of entanglements. In this case,

$$
\left.C_{N}^{3}=\sqrt{\frac{N}{6(N-1)} \sum\left(\left|a_{i j k} a_{p q m}-a_{i q k} a_{p j m}\right|^{2}+\left|a_{i j k} a_{p q m}-a_{p j k} a_{i q m}\right|^{2}\right.}\right)<1 .
$$

For instance, for a system of two maximally entangled qubits and one separated qubit, $a_{111}=a_{222}=a_{112}=a_{221}=\frac{1}{2}$ and the rest $a_{i j k}=0$, we have $C_{N}^{3}=\sqrt{\frac{5}{6}}$. 
For $M N$-dimensional quantum systems,

$$
\Psi_{M}=\sum_{i_{1}, \ldots, i_{M}=1}^{N} a_{i_{1}, \ldots, i_{M}} e_{i_{1}} \otimes \ldots \otimes e_{i_{M}}
$$

$a_{i_{1}, \ldots, i_{M}} \in \mathbb{C}$, besides a quadratic invariant:

$$
I_{0}=\sum_{i_{1}, \ldots, i_{M}=1}^{N} a_{i_{1}, \ldots, i_{M}} a_{i_{1}, \ldots, i_{M}}^{*} \equiv 1
$$

there are biquadratic invariants of the form

$$
I_{\alpha \beta}=\sum a_{\alpha \beta} a_{\alpha \beta^{\prime}}^{*} a_{\alpha^{\prime} \beta^{\prime}} a_{\alpha^{\prime} \beta}^{*},
$$

where $\alpha$ and $\alpha^{\prime}$ (resp. $\beta$ and $\beta^{\prime}$ ) are subset of the subindices of $a$, associated to the same sub Hilbert spaces but with different summing indices. $\alpha$ (or $\alpha^{\prime}$ ) and $\beta$ (or $\beta^{\prime}$ ) span the whole space of a given subindex of $a$.

Under local unitary transformation, $a_{i_{1}, \ldots, i_{M}}$ is mapped to $\sum a_{j_{1}, \ldots, j_{M}} b_{j_{1} i_{1}}^{1} \ldots b_{j_{M} i_{M}}^{M}$, with $b_{j_{k} i_{k}}^{k}, k=1, \ldots, M$, standing for the unitary transformation on $k$-th quantum space, $\sum_{l=1}^{N} b_{j l}^{k} b_{j^{\prime} l}^{k *}=$ $\delta_{j j^{\prime}}$. It is straightforward to check that under this transformation $I_{\alpha \beta}$ is an invariant.

From (18) and (19) we have

$$
I_{0}^{2}-I_{\alpha \beta}=\frac{1}{2} \sum_{\left\{\alpha, \alpha^{\prime}, \beta, \beta^{\prime}\right\}}^{N}\left|a_{\alpha \beta} a_{\alpha^{\prime} \beta^{\prime}}-a_{\alpha \beta^{\prime}} a_{\alpha^{\prime} \beta}\right|^{2} .
$$

Altogether we have $d=2^{M-1}-1$ biquadratic invariants, corresponding to different selections of the sub index sets of $\alpha, \beta$. The generalized concurrence is then given by

$$
C_{N}^{M}=\sqrt{\frac{N}{d(N-1)}\left(d I_{0}^{2}-I_{1}-\ldots-I_{d}\right)}=\sqrt{\frac{N}{2 d(N-1)} \sum_{p} \sum_{\left\{\alpha, \alpha^{\prime}, \beta, \beta^{\prime}\right\}}^{N}\left|a_{\alpha \beta} a_{\alpha^{\prime} \beta^{\prime}}-a_{\alpha \beta^{\prime}} a_{\alpha^{\prime} \beta}\right|^{2}}
$$

where $\sum_{p}$ stands for the summation over all possible combinations of the indices of $\alpha$ and $\beta$. From (20) the separability conditions for multipartite mixed states can be studied [25].

We have studied the quantum entanglements for $N$-dimensional bipartite quantum systems and multiparticle systems in terms of of invariants under local unitary transformations. Using the properties of the generalized concurrence the entanglement of formation and the separability of high dimensional mixed states can be investigated. 
ACKNOWLEDGEMENTS: We would like to thank C. Bennet, L.M. Duan, L.D. Gottesman, H.K. Lo, R.F. Werner and W.K. Wootters for useful discussions and communications.

\section{References}

[1] J. Bell, Physics 1 (1964) 195.

[2] See, for example, D.P. DiVincenzo, Science 270, 255 (1995).

[3] C.H. Bennett, G. Brassard, C. Crépeau, R. Jozsa, A. Peres, and W.K. Wootters, Phys.Rev.Lett. 70, 1895 (1993).

[4] S. Albeverio and S.M. Fei, Phys.Lett. A 276(2000)8-11.

[5] C.H. Bennett and S.J. Wiesner, Phys.Rev.Lett. 69, 2881 (1992).

[6] See, for example, C.A. Fuchs, N. Gisin, R.B. Griffiths, C-S. Niu, and A. Peres, Phys.Rev.A, 56, 1163 (1997) and references therein.

[7] C.H. Bennett, D.P. DiVincenzo, J.A. Smolin, and W.K. Wootters, Phys. Rev. A 54, 3824 (1996).

[8] C.H. Bennett, H.J. Bernstein, S. Popescu, and B. Schumacher, Phys. Rev. A 53, 2046 (1996).

[9] V. Vedral, M.B. Plenio, M.A. Rippin, and P.L. Knight,Phys. Rev. Lett. 78, 2275 (1997);

V. Vedral, M.B. Plenio, K. Jacobs, and P.L. Knight, Phys. Rev. A 56, 4452 (1997);

V. Vedral and M.B. Plenio, Phys. Rev. A 57, 1619 (1998).

[10] A. Peres, Phys. Rev. Lett. 761413 (1996).

[11] K. Życzkowski and P. Horodecki, Phys. Rev. A 58, 883 (1998).

[12] B. Schumacher and M.D. Westmoreland, Relative entropy in quantum information theory, quant-ph/0004045.

[13] M. Horodecki, P. Horodecki, and R. Horodecki, Phys. Rev. Lett. 80, 5239 (1998). 
[14] S. Hill and W.K. Wootters, Phys. Rev. Lett. 78, 5022 (1997).

W.K. Wootters, Phys. Rev. Lett. 80, 2245 (1998).

[15] R.F. Werner and M.M. Wolf, Bell's inequalities for states with positive partial transpose, quant-ph/9910063.

[16] L. Henderson and V. Vedral, Phys. Rev. Lett. 84, 2263 (2000).

M. Horodecki, P. Horodecki and R. Horodecki, Phys. Rev. Lett. 84, 2014 (2000).

[17] N. Linden and S. Popescu, Fortsch. Phys. 46 (1998)567-578.

[18] A. Peres, Phys. Rev. Lett. 77, 1413 (1996).

[19] M. Horodecki, P. Horodecki, and R. Horodecki, Phys. Lett. A 223, 1 (1996).

[20] M. Lewenstein, D. Bruss, J. I. Cirac, B. Kraus, M. Kuś, J. Samsonowicz, A. Sanpera, and R. Tarrach, J. Mod. Opt. 47, 2481 (2000).

[21] M. Horodecki, P. Horodecki, and R. Horodecki, preprint LANL quant-ph/0006071

[22] S. Albeverio, S.M. Fei and D. Goswami, Separability of Rank Two Quantum States, SFB-256 preprint, 2001.

[23] P. Horodecki, J.A. Smolin, B.M. Terhal and A.V. Thapliyal, Rank two bipartite bound entangled states do not exist, quant-ph/9910122.

[24] S.M. Fei, J. Jost and G.F. Wang, Entanglement of Formation for a Class of Quantum States, MIS preprint, 2001.

[25] S. Albeverio, S.M. Fei and D. Goswami, Separability of Multipartite Quantum States, in preparation. 